\title{
Microbial Communities in Different Tissues of Atta sexdens rubropilosa Leaf-cutting Ants
}

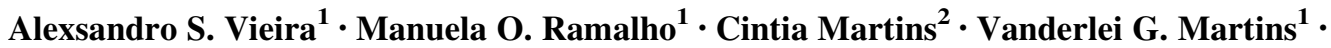 \\ Odair C. Bueno ${ }^{1}$
}

Received: 6 February 2017/ Accepted: 11 July 2017/Published online: 18 July 2017

(C) Springer Science+Business Media, LLC 2017

\begin{abstract}
Bacterial endosymbionts are common in all insects, and symbiosis has played an integral role in ant evolution. Atta sexdens rubropilosa leaf-cutting ants cultivate their symbiotic fungus using fresh leaves. They need to defend themselves and their brood against diseases, but they also need to defend their obligate fungus gardens, their primary food source, from infection, parasitism, and usurpation by competitors. This study aimed to characterize the microbial communities in whole workers and different tissues of $A$. sexdens rubropilosa queens using Ion Torrent NGS. Our results showed that the microbial community in the midgut differs in abundance and diversity from the communities in the postpharyngeal gland of the queen and in whole workers. The main microbial orders in whole workers were Lactobacillales, Clostridiales, Enterobacteriales, Actinomycetales, Burkholderiales, and Bacillales. In the tissues of the queens, the main orders
\end{abstract}

\author{
Alexsandro S. Vieira \\ alexsvieira@yahoo.com.br \\ Manuela O. Ramalho \\ manuramalho2010@gmail.com \\ Cintia Martins \\ martins.c@ufpi.edu.br \\ Vanderlei G. Martins \\ martv@uol.com.br \\ Odair C. Bueno \\ odaircb@rc.unesp.br \\ 1 Centro de Estudos de Insetos Sociais, UNESP - Univ \\ Estadual Paulista, Campus Rio Claro, Av. 24A, 1515, Bela \\ Vista, Rio Claro, São Paulo 13506-900, Brazil \\ 2 Universidade Federal do Piauí - Campus Ministro Reis \\ Velloso, Av. São Sebastião, 2819, Parnaíba, \\ Piauí 64.202-020, Brazil
}

were Burkholderiales, Clostridiales, Syntrophobacterales, Lactobacillales, Bacillales, and Actinomycetales (midgut) and Entomoplasmatales, unclassified $\gamma$-proteobacteria, and Actinomycetales (postpharyngeal glands). The high abundance of Entomoplasmatales in the postpharyngeal glands $(77 \%)$ of the queens was an unprecedented finding. We discuss the role of microbial communities in different tissues and castes. Bacteria are likely to play a role in nutrition and immune defense as well as helping antimicrobial defense in this ant species.

Keywords Attini - Endosymbiont - Entomoplasmatales · Next-generation sequencing

\section{Introduction}

Ants (Hymenoptera: Formicidae) represent a third of the insect biomass in the tropical rainforests of South America, with more than 13,000 species separated into different evolutionary branches that have had great ecological success [2, 19]. Symbiosis can be considered one of the driving forces in ant evolution and diversification $[3,28,37]$. Over the course of their 115-168-million-year history [7, 28], these diverse and ecologically dominant insects have repeatedly evolved symbiotic relationships with sap-feeding insects [40], plants [17], and microbes $[5,29,56]$. For instance, fungus-growing attines have obligate and ancient mutualisms with basidiomycete fungi (approximately 50 million years BP [30, 41]); not only do these ants defend themselves and their brood against diseases, but they also defend their primary food source, obligate fungus gardens, from infection, parasitism, and usurpation by competitors [18]. 
Endobacteria may play a role in the host's biological system. For example, carpenter ants harbor Blochmannia species that are responsible for nitrogen recycling and nutrient upgrading [13]. However, interactions between bacteria and herbivorous and fungivorous ants have not been extensively explored, although they are thought to have played a key role in the success of these ants [5, 37]. Actinomycetes (phylum Actinobacteria) are the beststudied bacterial symbionts of the higher fungus-growing ants. One example is genus Pseudonocardia, which produces antibiotics against numerous bacterial and fungal microbes [31, 36, 42, 57]. These metabolites are narrowspectrum antimicrobials that are active against Escovopsis, a potentially virulent, specialized fungus that attacks the ants' mutualistic fungus [7, 8]. Several other bacterial symbionts may be involved in the defense against parasites and diseases, in addition to having other roles. Examples include Streptomyces species associated with Acromyrmex octospinosus [4, 20, 44, 45], Amycolatopsis in Mycocepurus smithii workers [42], and Burkholderia in fungus gardens of A. sexdens rubropilosa [46]. Atta and Acromyrmex are derived attines and present the well-developed metapleural gland in relation to basal fungusgrowing attines [52, 53]; this gland produces antibiotic compounds that inhibit pathogens. However, which bacteria would be assisting the antimicrobial defense of $A$. sexdens rubropilosa?

Only a few studies used next-generation sequencing (NGS) techniques to study microbial communities in fungus-growing ants. Kellner et al. [24] used pyrosequencing to describe the bacterial community in M. smithii, a lower fungus-growing ant, and found a low abundance of actinomycete bacteria. Instead, the bacterial community was characterized by a high abundance of Lactobacillus and Pantoea, both in the garden and in worker ants. In Trachymyrmex septentrionalis ants, 454 sequencing revealed a diverse group of associated Actinobacteria, most notably Solirubrobacter, Pseudonocardia, and Microlunatus [22]. Meirelles et al. [32] used Illumina sequencing to study the microbiome in workers of the leaf-cutter ant Atta texana, and found that Pseudomonas, Acinetobacter, Propionibacterium and Corynebacterium were consistently abundant, and that some pellet and ant samples had a high abundance of Mesoplasma bacteria (clade Entomoplasmatales).

Sapountzis et al. [47] found that Acromyrmex leaf-cutting ants have simple gut microbiota (Rhizobiales) with nitrogen-fixing potential. In non-fungus-growing ants, several symbiotic bacteria grow in the ant gut, which is inhabited by a wide range of microorganisms $[1,23,26,37,48]$. The composition of the gut bacterial community varies among ant taxa and across trophic levels [11], as well as between tissues [26]. In the Sonoran Desert turtle ant Cephalotes rohweri, for instance, the proventriculus functions as a micropore filter, blocking the entry of bacteria and particles $\geq 0.2 \mu \mathrm{m}$ into the midgut and hindgut, while allowing dissolved nutrients through, thus protecting the gut microbiome from disruption [26]. As a result, different parts of the digestive system have different bacterial communities.

Next-generation sequencing is an excellent tool to characterize the microbial communities associated with insects. This study aimed to characterize the microbial communities in whole workers and different tissues of the queen of A. sexdens rubropilosa using Ion Torrent NGS. The queens were chosen for the study because they belong to the reproductive caste and are responsible for vertical microbiome transmission, whereas the workers were chosen because they belong to a sterile caste. This study of the microbiome may provide answers on the ability of fungi-forming ants to control pathogens since we already know that the main function of the metapleural gland is to produce antibiotic compounds, but little is known about how bacteria could contribute to the defense antimicrobial activity of ants. We hypothesized that whole workers and queens would have different microbial communities, and that the community in the midgut would differ from the community in the postpharyngeal gland of the foregut; this gland was thought to be exclusive to Formicidae [11], but Herzner and collaborators [18] found them in the solitary wasps. Recently, Decio and collaborators [10] defined this gland as a diverticulum of the anterior intestine with the ability to absorb, store, metabolize, and mobilize lipids to the hemolymph [54], so this study investigated whether PPG, which belongs to the digestive tract, differs from its microbial communities in relation to the midgut.

\section{Materials and Methods}

Three whole workers from three different colonies were collected to create a DNA pool. In addition, the queens from the same three colonies were dissected to extract the midgut (MD) and postpharyngeal gland (PPG) to create two additional DNA pools. All colonies were collected from a pine reforestation area in Itirapina, SP, Brazil (S $22^{\circ} 14^{\prime} 42^{\prime \prime}$, W $47^{\circ} 50^{\prime} 34^{\prime \prime}$ ). Individuals were placed in $95 \%$ ethanol until further processing.

\section{DNA Extraction and Sequencing}

For DNA extraction, all specimens (workers and queens) were anesthetized by cooling $\left(4^{\circ} \mathrm{C}\right)$ and subjected to ultrasonic cleaning for $1 \mathrm{~min}$. The queens were dissected in a Petri dish containing an insect saline solution $(0.128 \mathrm{M}$ 
$\mathrm{NaCl}, 0.016 \mathrm{M} \mathrm{Na}_{2} \mathrm{HPO}_{4}, 0.019 \mathrm{M} \mathrm{KH}_{2} \mathrm{PO}_{2}, \mathrm{pH}$ 7.2); the $\mathrm{MD}$ and PPG were removed under a stereomicroscope.

Total DNA pools were extracted from: (a) whole workers (WW), (b) the midguts of the queens (MD), and (c) the postpharyngeal glands of the queens (PPG). Dissected organs were sonicated and incubated at $55^{\circ} \mathrm{C}$ for $3 \mathrm{~h}$ in a lysis solution with addition of proteinase $\mathrm{K}$ (100 mM Tris at pH 9.1, $100 \mathrm{mM} \mathrm{NaCl}, 200 \mathrm{mM}$ sucrose, $50 \mathrm{mM}$ EDTA, 0.5\% SDS). Protein residues were precipitated with $5 \mathrm{M} \mathrm{NaCl}$, and the DNA was subjected to ethanol precipitation and $70 \%$ ethanol wash. The ethanol was removed and the DNA pellet was dried overnight in the dark. The DNA was hydrated in $15 \mu \mathrm{L}$ of TE buffer (10 mM Tris, 1 mM EDTA, pH 8) [39]. Total DNA pools were sent to Life Technologies for sequencing on the Ion Torrent PGM (Personal Genome Machine).

The V4 region of the 16S rRNA gene was amplified from approximately $20.6 \mathrm{ng}, 16.8 \mathrm{ng}$, and $13.9 \mathrm{ng}$ of total WW, MD and PPG DNA pools, respectively, resulting in a 209-bp fragments. The PCR was carried out using $1.5 \mathrm{U}$ Platinum Taq DNA Polymerase, $1 \mathrm{X}$ buffer, $0.2 \mathrm{mM}$ dNTP, $2.5 \mathrm{mM} \mathrm{MgCl}_{2}$ (New England Biolabs, UK), and primers 515F (5'-GTGCCAGCMGCCGCGGTAA-3') and 806R (5'-GGACTACHVGGGTWTCTAAT-3') [9] with the following cycling conditions: $94{ }^{\circ} \mathrm{C}$ for $5 \mathrm{~min} ; 30$ cycles at $94{ }^{\circ} \mathrm{C}$ for $30 \mathrm{~s}, 54{ }^{\circ} \mathrm{C}$ for $30 \mathrm{~s}, 72{ }^{\circ} \mathrm{C}$ for $1 \mathrm{~min}, 72{ }^{\circ} \mathrm{C}$ for 5 min. Amplicons were purified using $2 \% \mathrm{E}-G e l^{\circledR}$ SizeSelect $^{\mathrm{TM}}$ agarose gel, and concentrated with $1.2 \times$ AMPure XP Beads (Beckman Coulter). Emulsion PCR was carried out using Ion OneTouch $2^{\mathrm{TM}}$ with the Ion PGM ${ }^{\mathrm{TM}}$ Template OT2 $400 \mathrm{Kit}$ (Life Technologies), following the manufacturer's instructions. Amplified products from each sample were barcoded and sequenced on the 314 semiconductor chip using Ion Torrent PGM (Life Technologies, CA, USA).

\section{Analyses of Microbial Diversity}

All reads obtained from WW, MD, and PPG were first filtered by fragment quality and size using the initial data processing tool available from the Ribosomal Database Project (RDP) server (http://pyro.cme.msu.edu/init/form). We used minimum expected average quality score of 20 and a minimum sequence length of $100 \mathrm{bp}$. All sequences that did not reach this score and length were discarded. We used UCHIME de novo [12] to detect chimeric sequences. Filtered sequences were aligned with the RDP Align tool and clustered into operational taxonomic units (OTUs) at different distance levels using the RDP Complete Linkage Clustering tool. The OTUs were identified upon comparison with sequences in the RDP server. The read counts of the three metabarcoding samples were normalized (transformed and standardized) using the MG-RAST analysis tool, so that the values of all samples were rescaled from 0 (minimum value) to 1 (maximum value). This is important for meaningful comparisons between samples. The RDP server was also used to estimate richness and diversity indices (Chaol, Shannon, and evenness) and to conduct rarefaction analyses at each dissimilarity level (Fig. 1 shows 3\% dissimilarity level). The resulting high-quality reads were used for subsequent analyses. The MG-RAST database v3.2 [33] was used to generate plots of the distribution of phyla and orders, and a heatmap, and to assign reads to taxonomic categories using RDP as a reference. Using the MG-RAST software, we created a tree diagram to analyze the diversity of bacterial phyla and orders found in the present study, and their distributions in each microbial community. The tree diagram allows the comparison of samples against a taxonomy hierarchy available from NCBI. The hierarchy is displayed as a rooted tree and the abundance (normalized for dataset size) for each dataset in the various categories is displayed as a bar chart for each category (Fig. 4). Each bacterial order is color-coded based on its phylum; some of the highly diverse orders are highlighted below. Metabarcoding sequence reads were submitted to MG-RAST under accession numbers 4689130.3, 4689135.3, and 4689142.3.

\section{Results}

In total, we obtained 498.288 reads (104 MB). Of these, 274.664 reads came from whole workers (WW), and 185.091 and 32.507 reads were from the midgut (MD) and postpharyngeal glands (PPG) of the queens, respectively. After samples were filtered for quality control, there were 182.732 (WW), 119.870 (MD), and 25.977 (PPG) reads left, confirming the success of the sequencing protocol. The microbiota communities associated with queens and workers from colonies of the leaf-cutting ant $A$. sexdens rubropilosa did not differ at the level of bacterial phylum. However, the relative abundance and diversity of bacterial orders varied between whole individuals and queen tissues.

\section{Rarefaction Analysis}

Rarefaction curves were used to assess the adequacy of the sampling effort for the analysis of microbial diversity. We chose a dissimilarity level of 0.03 for the rarefaction curves. The WW and MD rarefaction curves did not completely plateau (Fig. 1), but their low slopes at higher sampling depths suggest that our sequencing effort was sufficient to identify all the major bacteria. 


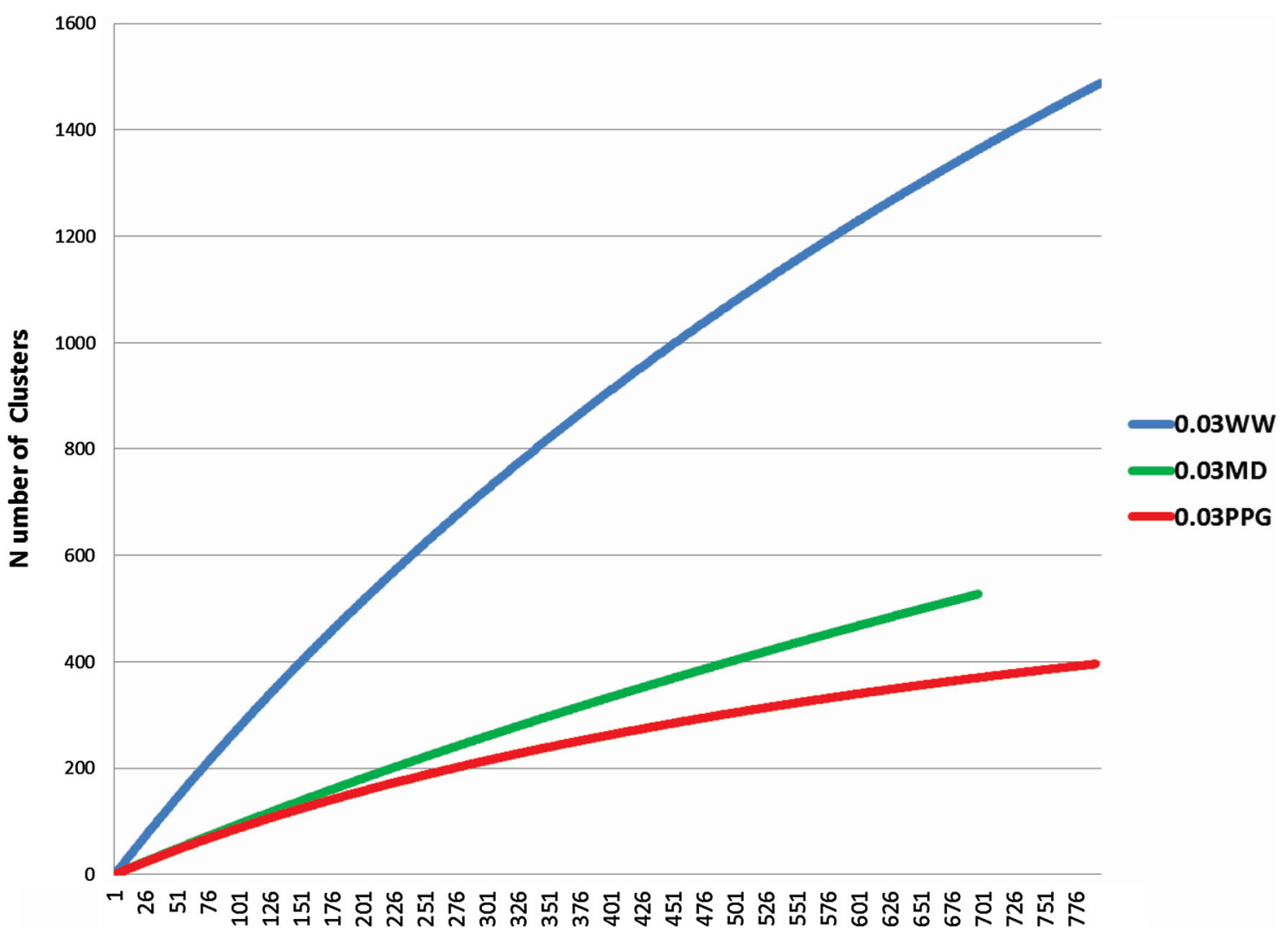

Fig. 1 Rarefaction curves used to estimate richness and sampling effort (3\% dissimilarity level). The $y$-axis shows the expected species count after sampling the number of sequences on the $x$-axis. $W W$ whole worker, $M D$ midgut, $P P G$ postpharyngeal gland

Table 1 Microbial diversity is based on the 16S rRNA gene library, at 3 and $5 \%$ dissimilarity levels

\begin{tabular}{lllllll}
\hline Sample & Reads & Distance units & OTU & Chao1 & Shannon $\left(\mathrm{H}^{\prime}\right)$ & Evenness (E) \\
\hline Whole worker & \multirow{2}{*}{274.664} & 0.00 & 2337 & 87.622 & 774.699 & 0.99876 \\
& & 0.03 & 1488 & 2.877 & 713.124 & 0.97619 \\
\multirow{3}{*}{ Midgut } & 0.05 & 921 & 1.334 & 649.924 & 0.95221 \\
& \multirow{2}{*}{185.091} & 0.00 & 693 & 48.096 & 653.883 & 0.99966 \\
& & 0.03 & 528 & 1.617 & 614.479 & 0.98017 \\
PPG & 0.05 & 353 & 548 & 560.187 & 0.9549 \\
& \multirow{3}{*}{32.507} & 0.00 & 749 & 10.229 & 659.256 & 0.99604 \\
& & 0.03 & 396 & 587 & 573.992 & 0.95963 \\
& & 0.05 & 235 & 334 & 504.542 & 0.92414 \\
\hline
\end{tabular}

\section{Estimates of Microbial Diversity}

The diversity of bacterial communities from different samples was estimated using several indices (Chaol, Shannon, and evenness) based on different distance units; using distance units at 0.03 the Chao1 indices were 2.877, 1.617 , and 587 to $\mathrm{WW}, \mathrm{MD}$, and PPG, respectively. Shannon indices were $713.124,614.479$, and 573.992 to WW, MD, and PPG, respectively. Evenness indices were $0.97619,0.98017$, and 0.95963 to $\mathrm{WW}, \mathrm{MD}$, and PPG, respectively (Table 1). As expected, WW samples had the highest diversity, followed by MD and PPG.

\section{Heatmap and Principal Component Analysis (PCA)}

A heatmap was constructed based on WW, MD, and PPG metabarcoding (Fig. 2a). Data were compared to the Ribosomal Database Project (RDP) database using an e-value threshold of $1 \times 10^{-5}$, a minimum identity of $97 \%$, and a minimum alignment length of $100 \mathrm{bp}$. The heatmap 


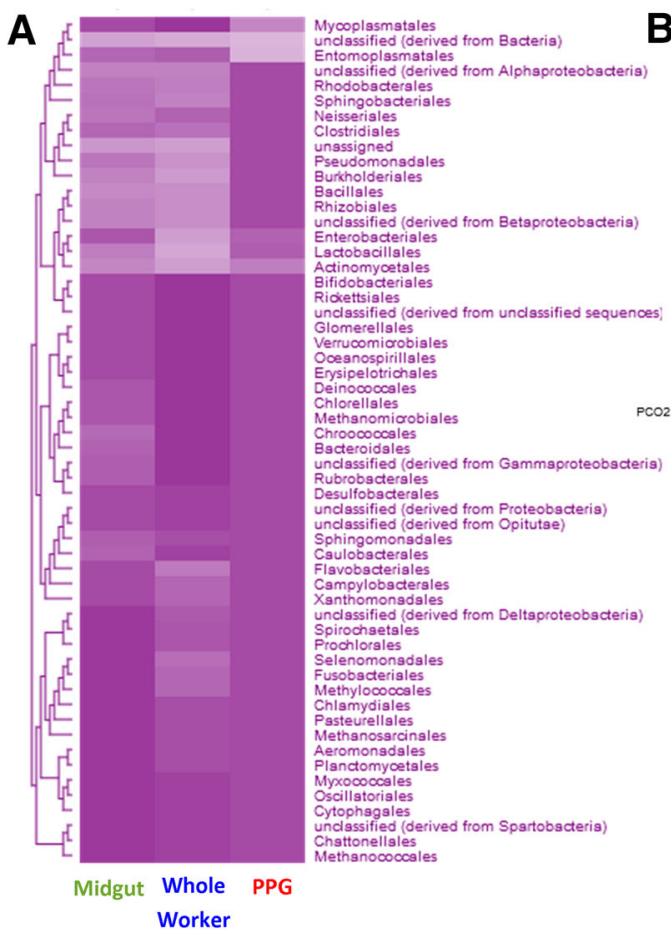

B

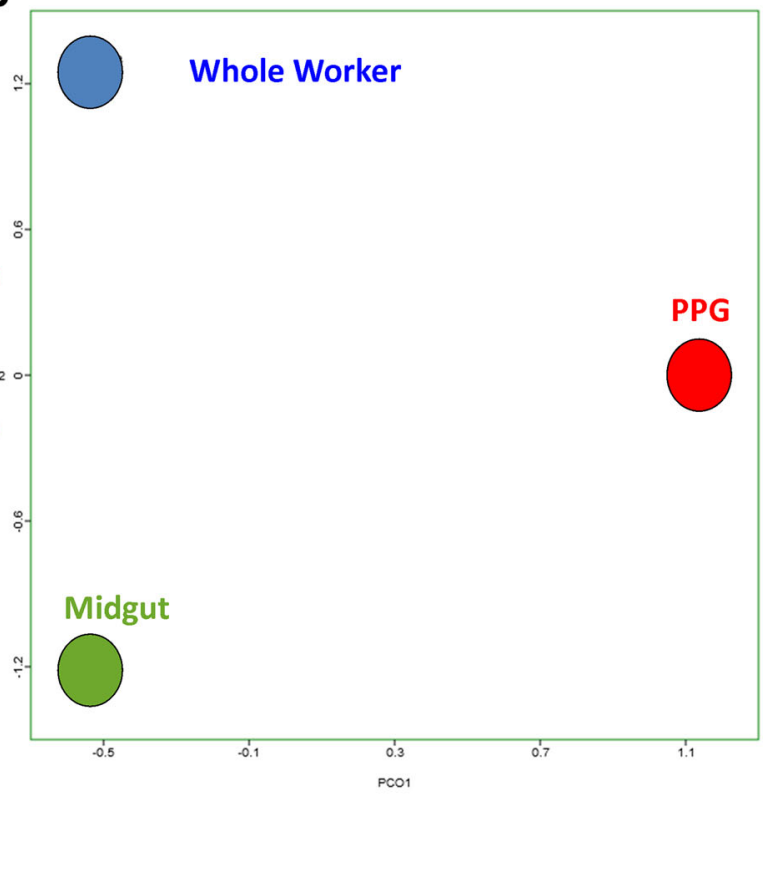

Fig. 2 Comparative bacterial communities in the leaf-cutting ant $A$. sexdens rubropilosa. a Heatmap showing significant variation in the relative abundances of different phyla of bacteria in whole workers (WW), queen midgut (MD), and queen postpharyngeal gland (PPG).

b Metagenome PCA (WW, MD, and PPG). Note that although some bacteria are shared between the WW, MD, and PPG metagenomes, these samples clustered separately in the PCA plot due to differences in their abundance profiles

was created using Ward's clustering of Bray-Curtis distances and grouped by order. The heatmap revealed significant variation in the relative abundances of different bacterial phyla in WW, MD, and PPG (Fig. 2a).

The PCA plot generated by the MG-RAST tool (BrayCurtis distances, grouped by order) shows that microbial community composition of WW, MD, and PPG are similarly different from each other (Fig. 2b). These data reveal that the individual datasets for each sample type are different; that is, each bacterial community in WW, MD, and PPG is clearly separated from the others in the PCA (Fig. 2b).

\section{Taxonomic Composition and Distribution of the Microbial Community at the Order Level}

Relative abundances of the bacterial orders varied according to sample type, as summarized in Fig. 3. In the WW DNA pool, the most abundant order was Lactobacillales (28\%), followed by Clostridiales (17\%), Enterobacteriales (11\%), Actinomycetales (7\%), Burkholderiales (5\%), and Bacillales (5\%), among others (Fig. 3). In the queen MD sample the most abundant orders were Burkholderiales (14\%), Clostridiales (10\%), Syntrophobacterales (8\%),

Lactobacillales (7\%), Bacillales (7\%), and Actinomycetales (6\%) (Fig. 3). Interestingly, both in WW and in MD the abundance of Entomoplasmatales order was less than $1 \%$. In contrast, in the queen PPG DNA pool the abundance of Entomoplasmatales was $77 \%$, followed by unclassified Gammaproteobacteria (14\%) and Actinomycetales (9\%) (Fig. 3).

\section{Taxonomic Composition and Distribution of the Microbial Community at the Phylum Level}

Examining the tree diagram, it is evident that the WW sample had the highest diversity of bacterial phyla, followed by the MD and PPG of the queens (Fig. 4). Phylum Actinobacteria was found in all three groups (WW, MD, and PPG; Fig. 4). Within this phylum, order Actinomycetales, which is phylogenetically close to Burkholderiales and Bifidobacteriales, was abundant in all samples. In contrast, phylum Bacteroidetes was present only in the MD and WW samples, and phyla Deinococcus-Thermus and Firmicutes were primarily found in WW and MD. Phylum Proteobacteria was more abundant in WW, followed by MD; both Burkholderiales and Enterobacteriales were abundant. Phylum Tenericutes was identified in all three 


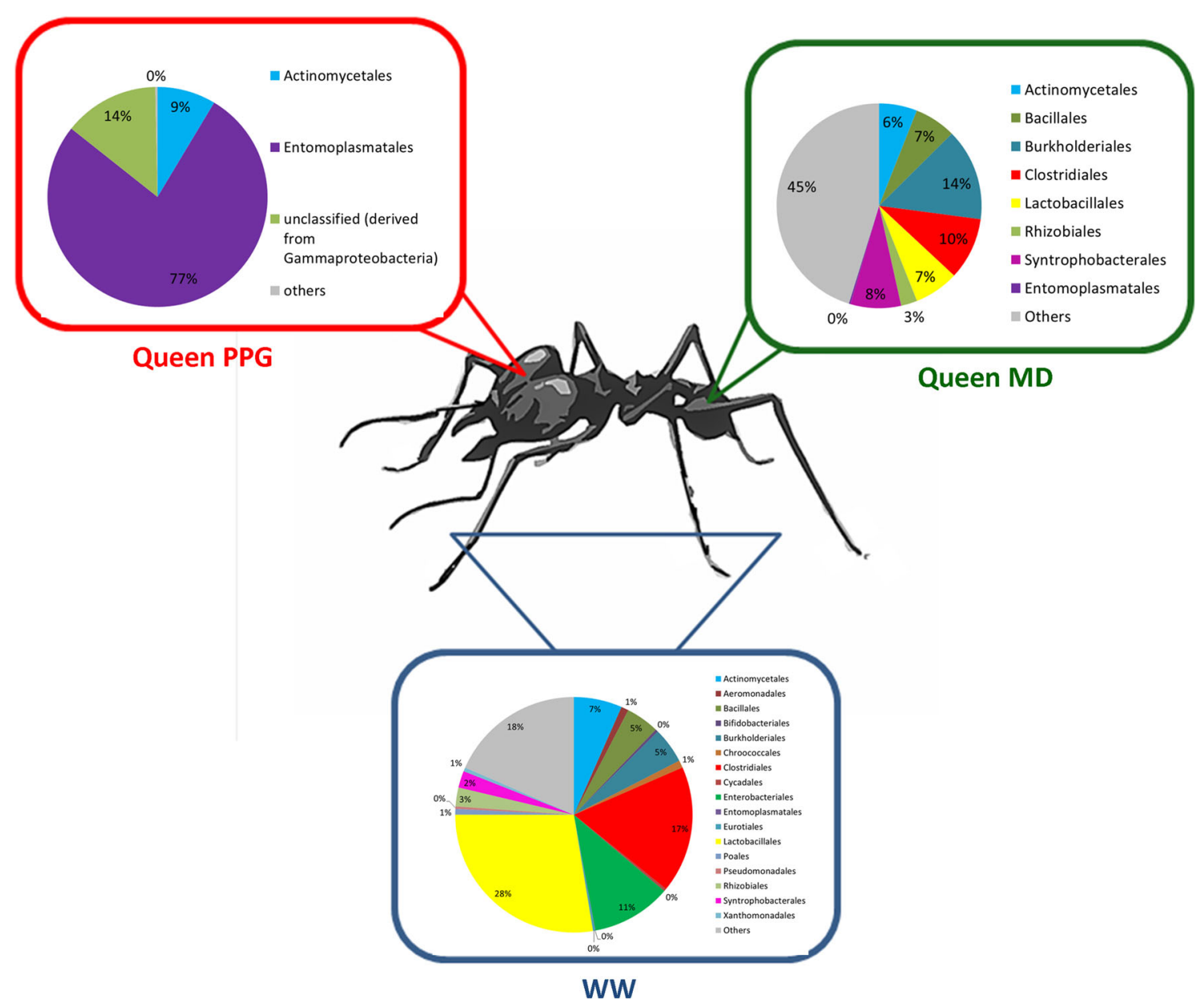

Fig. 3 Comparative taxonomic composition and distribution of the bacterial community associated in different organs of queens and workers of A. sexdens rubropilosa at the level of order. WW, blue rectangle $=$ Relative abundances in the bacterial community of

groups, but it was more common in PPG samples, represented by orders Entomoplasmatales and Mycoplasmatales (Fig. 4).

\section{Discussion}

Our study was the first to identify the microbial communities in different tissues of the leaf-cutting ant $A$. sexdens rubropilosa and to use metabarcoding to describe its high diversity. Our results also showed that the microbial communities in whole workers and in the midgut and the postpharyngeal gland of the queens all differed in abundance and diversity. Some authors have proposed that herbivores may need more bacteria than omnivores and predators, which might explain the high bacterial diversity associated with $A$. sexdens rubropilosa, a generalist herbivore. For instance, bacterial diversity in Cephalotes, a herbivorous ant genus, is whole workers; $\mathrm{MD}$, green rectangle $=$ Relative abundances in the bacterial communities in the midgut of queens; PPG, red rectangle $=$ Relative abundances in the bacterial communities in the postpharyngeal glands of queens (Color figure online)

greater than in predatory ant species [49]. Our sample sizes were small, but our results are unprecedented; this is the first research to study the microbial community for this species and these tissues. We chose to focus only on certain orders of bacteria sampled with high abundance from the MD and PPG tissues, and WW.

The main phyla in all samples were Actinobacteria, Bacteroidetes, Deinococcus-Thermus, Firmicutes, Proteobacteria, and Tenericutes. These bacterial communities were similar to the communities in A. texana queens [32]; the two species differed only in Deinococcus-Thermus. The similarity between the microbiomes of the two species of leaf-cutting ants in different environments, A. texana from North America and A. sexdens rubropilosa from South America, suggests that similar ecology breeds similar bacterial communities. Another suggestion would be stem from a common ancestor of both, but others studies are necessary to confirm this. 


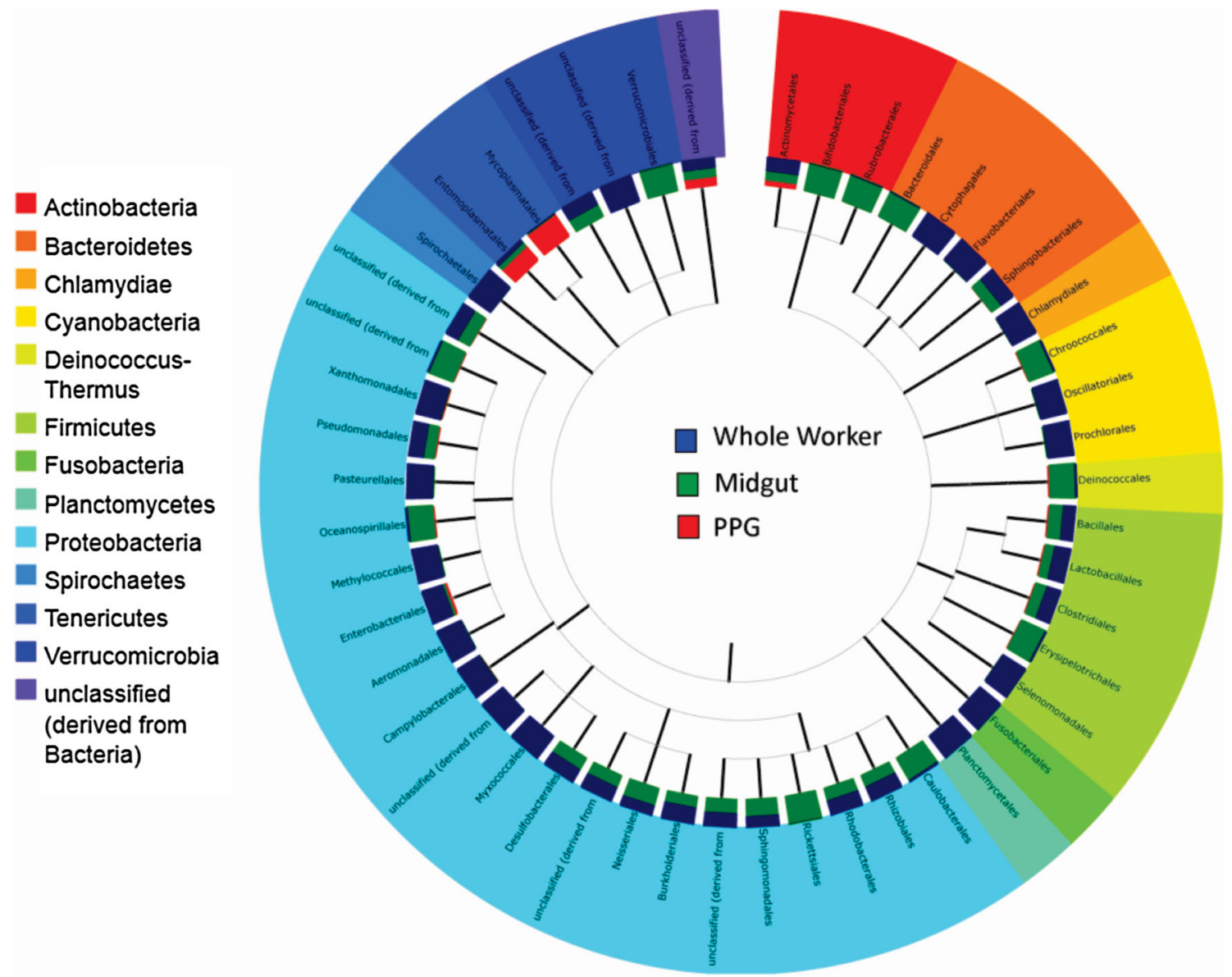

Fig. 4 Comparative tree diagram of phyla and orders of the bacterial communities in whole workers and the midgut and postpharyngeal glands of $A$. sexdens rubropilosa queens

There was greater similarity, in phylum, between WW and MD compared with PPG (Fig. 3). In termite guts, variation in the structure of bacterial communities may be explained by variation in physiological conditions, such as $\mathrm{pH}[15,34]$, while in Cephalotes ants the proventriculus acts as a bacterial filter [26]. Similar morphological and physiological barriers could contribute to the abundance and diversity of the bacterial communities in the gut and PPG tissues in the present study. Alternatively, differences in microbial diversity may be related to the roles of different castes. While the sterile worker caste is responsible for maintaining the fungus garden and caring for immatures, the queen caste is responsible for reproduction. However, allogrooming among colony individuals could allow horizontal transmission of bacteria between castes and may thus render the microbial communities similar.

Abundance and diversity of the MD microbiome differs from that of PPG. These differences in the microbiome can be explained by morphological structures that could be filtering the passage of strains of different sizes, for example Lanan and collaborators [26] in Cephalotes ants shows that the proventriculus is a bacterial filter; here we suggest that there is also a small gap between the pharynx and the PPG that can filter out certain bacteria and therefore could explain different diversity in these organs in the digestive tract.

Phylum Actinobacteria (order Actinomycetales) was present in WW (7\%), MD (6\%), and PPG (9\%). Actinomycetales was also found through pyrosequencing in workers of the basal fungus-growing ant Mycocepurus smithii [24]. Actinomycetes bacteria that the ants culture on their cuticles and antimicrobial compounds produced by themselves are thought to be specifically active against Escovopsis [6, 7, 14]. However, theses bacteria may produce antibiotics that contribute to the immune defense of $A$. sexdens rubropilosa ants together with the metapleural gland against pathogens. Their presence in the MD and PPG could result from the feeding habits of these ants, since these bacteria are also found in the fungus garden, or from grooming, which would allow the strains to reach these tissues.

Phylum Bacteroidetes was found only in the MD tissues of queens and WW. This phylum is abundant in the gardens of fungus-growing ants $[42,43]$, and may be involved in 
carbohydrate degradation. Phylum Deinococcus-Thermus was relatively rare in WW, MD, and PPG. This group of bacteria is highly hazard-resistant; Deinococcus sp. has only been recorded in extreme environments, such as hot springs and radioactive waste disposal sites [35].

Phylum Firmicutes was very abundant, especially order Lactobacillales, a pattern that is similar to that of previous studies on workers of the basal fungus-growing ant $M$. smithii [24], the turtle ant Cephalotes rohweri [26], and several species of Polyrhachis [38]. Species in genus Lactobacillus are well-known commensals of humans, insects, and other animals and often have antimicrobial properties [21]. This protective role has also been suggested for Lactobacillus in fungus-growing termites [27]. Although metapleural glands, which are exclusive to ants, also synthesize substances with antibiotic properties $[52,53]$, and the presence of Lactobacillales bacteria (28\%) in workers suggests the existence of an alternative defense mechanism. Lactobacillales bacteria may assist $A$. sexdens rubropilosa in defending fungus gardens against microorganisms, contributing to ant immunity. In addition, their presence inside the MD and PPG could result in the production of acids that change the $\mathrm{pH}$ of these environments and prevent infections by other agents [24].

Proteobacteria have previously been observed in the fungus gardens of Atta cephalotes, A. colombica, and A. sexdens [43], in pellets and queens' body parts (head, thorax, abdomen) of A. texana [32], and in the gut of four species of Acromyrmex [47]. Suen et al. [50] determined that hemicellulose and cellulose degradation occurred throughout the fungus gardens of a leaf-cutting ant, while lignin content remained relatively unchanged. These gardens were dominated by Gram-negative bacteria (phyla Proteobacteria and Bacteroidetes), which contributed to the degradation of plant polymers. Although Alphaproteobacteria (Rhizobiales) species are strictly extracellular and confined to the gut lumen, in the hindgut these symbionts produce bacterial NifH proteins, which have been associated with the fixation of nitrogen [47]. Of all orders of proteobacteria, Burkholderiales was the most abundant in the MD (14\%). In the leaf-cutting ant A. sexdens rubropilosa, the antifungal agent secreted by Burkholderia inhibited the germination of Beauveria bassiana, Metarhizium anisopliae, Verticillium lecanii, and Escovopsis weberi [46]. Similarly, Burkholderiales in the MD of leafcutting ants may contribute to nutrition and to improve their immune defense, although this needs to be further investigated.

Phylum Tenericutes, represented primarily by Entomoplasmatales, was found in all samples of A. sexdens rubropilosa. A host-specific clade of this order was not only found in the digestive system of army ants, primarily associated with the gut, but also in eggs, larvae, and pupae, suggesting a nutritional role [16]. Similarly, Entomoplasmatales are intra- and extra-cellular (gut) symbionts of Acromyrmex leaf-cutting ants, and Sapountzis et al. [47] suggested that these bacteria may aid in chitin digestion. However, Entomoplasmatales can also be pathogenic to plants and vertebrates [51]. For instance, Kautz et al. [25] detected a Spiroplasma infection in a Cephalotes varians colony, considering it a potential pathogen. A surprising finding was the high abundance of Entomoplasmatales in the PPG (77\%) of A. sexdens rubropilosa queens. Recently, Decio et al. [10] described the PPG as a specialized organ for lipid nutrition, similar to a foregut diverticulum, with the ability to absorb, store, metabolize, and mobilize lipids to the hemolymph of adult $A$. sexdens rubropilosa workers. Thus, the high abundance of Entomoplasmatales in the PPG may play an auxiliary role in lipid nutrition, although this interaction of the lipid metabolism and the presence of Entomoplasmatales needs to be better investigated.

Phylum Verrucomicrobia was found in small amounts, and only in WW and in the queen MD of A. sexdens rubropilosa. However, the contribution of this phylum for cellulose degradation, nitrogen fixation, and conversion of plant polysaccharides to acetate in termite species is well documented [55]. Order Opitutales (phylum Verrucomicrobia) was observed in the arboreal ant Cephalotes [1], and may be involved in nitrogen cycling for reincorporation by the ant. Since leaf-cutting ants are also herbivorous, these bacteria could have the same role in the worker and queen castes.

In conclusion, our results showed that the microbial communities in the queen midgut differ in abundance and diversity from the community in the queen postpharyngeal gland and in whole workers of $A$. sexdens rubropilosa leafcutting ants. The bacteria in the digestive tissues of the queens of this ant species may play a role in their nutrition, as well as immune defense, helping antimicrobial defense in fungus-growing ants. Although bacteria in the same order may have the same function, they may also fulfill different roles in different niches. Their function may also differ between sterile and reproductive castes. Further research will be conducted to work out the details of this host-symbiont relationship.

Acknowledgements We are grateful to FAPESP (Fundação de Amparo à Pesquisa do Estado de São Paulo), Grant No. 2012/12541-3 to ASV, CNPq (Conselho Nacional de Desenvolvimento Científico e Tecnológico), Grants No. 157837/2015-7 to ASV and 306910/2011-0 to OCB, and CAPES (Coordenação de Aperfeiçoamento de Pessoal de Nível Superior), Grant No. 007343/2014-00) to MOR, for funding. We are grateful to CEIS (Center for the Study of Social Insects) at Universidade Estadual Paulista "Júlio de Mesquita Filho" (UNESP)Rio Claro, SP, Brazil, for granting access to the Molecular Biology Laboratory. We also thank Life Technologies (CA, USA) for sequencing using Next-generation sequencing (Ion Torrent PGM) equipments. 


\section{Compliance with Ethical Standards}

Conflict of interest The authors declare that they have no conflict of interest.

\section{References}

1. Anderson KE, Russell JA, Moreau CS et al (2012) Highly similar microbial communities are shared among related and trophically similar ant species. Mol Ecol 21:2282-2296. doi:10.1111/j.1365294X.2011.05464.x

2. Bolton B (2014) An online catalog of the ants of the world. http:// antcat.org. Accessed 01 Oct 2016

3. Brady SG, Fisher BL, Schultz TR et al (2006) Evaluating alternative hypotheses for the early evolution and diversification of ants. Proc Natl Acad Sci USA 103:18172-18177. doi:10.1073/ pnas.0605858103

4. Barke J, Seipke R, Gruschow S et al (2010) A mixed community of actinomycetes produce multiple antibiotics for the fungus farming ant Acromyrmex octospinosus. BMC Biol 8:109. doi:10. 1186/1741-7007-8-109

5. Cook SC, Davidson DW (2006) Nutritional and functional biology of exudate-feeding ants. Entomol Exp Appl 118:1-10. doi:10.1111/j.1570-7458.2006.00374.x

6. Currie CR (2001) A community of ants, fungi, and bacteria: a multilateral approach to studying symbiosis. Annu Rev Microbiol 55:357-380

7. Currie C, Scott J, Summerbell R et al (1999) Fungus-growing ants use antibiotic-producing bacteria to control garden parasites. Nature 398:701-705. doi:10.1038/19519

8. Currie CR, Poulsen M, Mendenhall J, Boomsma JJ, Billen J (2006) Coevolved crypts and exocrine glands support mutualistic bacteria in fungus-growing ants. Science 311:81-83. doi:10.1126/ science. 1119744

9. Caporaso JG, Lauber CL, Walters WA et al (2011) Global patterns of 16S rRNA diversity at a depth of millions of sequences per sample. PNAS 15:4516-4522. doi:10.1073/pnas.1000080107

10. Decio P, Vieira AS, Dias NB et al (2016) The postpharyngeal gland: specialized organ for lipid nutrition in leaf-cutting ants. PLoS ONE 11(5):e0154891. doi:10.1371/journal.pone.0154891

11. Delage-Darchen B (1976) Les glandes postpharyngeal dês fourmis connaissances actuellessur leur structure, leur fonctionnement, leur rôle. L'Année Biologique 15(1-2):63-76

12. Edgar RC, Haas BJ, Clemente JC, Quince C, Knight R (2011) UCHIME improves sensitivity and speed of chimera detection. Bioinformatics 27:2194-2200. doi:10.1093/bioinformatics/btr381

13. Feldhaar H, Straka J, Krischke M et al (2007) Nutritional upgrading for omnivorous carpenter ants by the endosymbiont Blochmannia. BMC Biol 5:48. doi:10.1186/1741-7007-5-48

14. Fernández-Marín H, Bruner G, Gomez EB et al (2013) Dynamic disease management in Trachymyrmex fungus-growing ants (Attini: Formicidae). Am Nat 181:571-582. doi:10.1086/669664

15. Fall S, Hamelin J, Ndiaye F et al (2007) Differences between bacterial communities in the gut of a soil-feeding termite $\mathrm{C}$ bitermes niokoloensis) and its mounds. Appl Environ Microbiol 73:5199-5208. doi:10.1128/AEM.02616-06

16. Funaro CF, Kronauer DJC, Moreau CS et al (2011) Army ants harbor a host-specific clade of Entomoplasmatales Bacteria. Appl Environ Microbiol 77:346-350. doi:10.1128/AEM.01896-10

17. Heil M, McKey D (2003) Protective ant-plant interactions as model systems in ecological and evolutionary research. Annu Rev Ecol Evol Syst 34:425-453. doi:10.1146/annurev.ecolsys.34. 011802.132410
18. Herzner G, Kaltenpoth M, Poettinger T et al (2013) Morphology, chemistry and function of the postpharyngeal gland in the South American digger wasps Trachypus boharti and Trachypus elongates. PLoS ONE 8(12):e82780. doi:10.1371/journal.pone. 0082780

19. Hölldobler B, Wilson EO (2011) The leafcutter ants. WW Norton, New York

20. Haeder S, Wirth R, Herz H et al (2009) Candicidin-producing Streptomyces support leaf-cutting ants to protect their fungus garden against the pathogenic fungus Escovopsis. Proc Natl Acad Sci USA 106:4742-4746. doi:10.1073/pnas.0812082106

21. Hammes WP, Vogel RF (1995) The genus Lactobacillus. In: Wood BJB, Holzapfel WH (eds) The genera of lactic acid bacteria. Chapman \& Hall, London, pp 19-54

22. Ishak HD, Miller JL, Sen R et al (2011) Microbiomes of ant castes implicate new microbial roles in the fungus growing ant Trachymyrmex septentrionalis. Sci Rep 1:204. doi:10.1038/ srep00204

23. Jaffe K, Caetano FH, Sánchez P et al (2001) Sensitivity of ant (Cephalotes) colonies and individuals to antibiotics implies feeding symbiosis with gut microorganisms. Can J Zool 79:1120-1124. http://hdl.handle.net/11449/33296

24. Kellner K, Ishak HD, Linksvayer TA et al (2015) Bacterial community composition and diversity in an ancestral ant fungus symbiosis. FEMS Microbiol Ecol 91:fiv073. doi:10.1093/femsec/ fiv073

25. Kautz S, Rubin BER, Moreau CS (2013) Bacterial Infections across the ants: frequency and prevalence of Wolbachia, Spiroplasma, and Asaia. Psyche. doi:10.1155/2013/936341

26. Lanan MC, Rodrigues PAP, Agellon A et al (2016) A bacterial filter protects and structures the gut microbiome of an insect. ISME J. doi:10.1038/ismej.2015.264

27. Long YH, Xie L, Liu N et al (2010) Comparison of gut-associated and nest-associated microbial communities of a fungus growing termite (Odontotermes yunnanensis). Insect Sci 17:265-276. doi:10.1111/j.1744-7917.2010.01327.x

28. Moreau CS, Bell CD, Vila R et al (2006) Phylogeny of the ants: diversification in the age of angiosperms. Science 312(5770):101-104. doi:10.1126/science.1124891

29. Mueller UG, Rehner SA, Schultz TR (1998) The evolution of agriculture in ants. Science 281:2034-2038. doi:10.1126/science. 281.5385.2034

30. Mueller UG (2002) Ant versus fungus versus mutualism: antcultivar conflict and the deconstruction of the attine ant-fungus symbiosis. Am Nat 160(Suppl. 4):S67-S98. doi:10.1086/342084

31. Mueller UG, Scott JJ, Ishak HD et al (2010) Monoculture of leafcutter ant gardens. PLoS ONE 5:e12668. doi:10.1371/journal. pone. 0012668

32. Meirelles LA, McFrederick QS, Rodrigues A et al (2016) Bacterial microbiomes from vertically transmitted fungal inocula of the leaf-cutting ant Atta texana. Environ Microbiol Rep 8(5):630-640. doi:10.1111/1758-2229.12415

33. Meyer F, Paarmann D, Souza MD et al (2008) The metagenomics RAST server-a public resource for the automatic phylogenetic and functional analysis of metagenomes. BMC Bioinform 9:386. doi:10.1186/1471-2105-9-386

34. Manjula A, Pushpanathan M, Sathyavathi S et al (2015) Comparative analysis of microbial diversity in termite gut and termite nest using ion sequencing. Curr Microbiol. doi:10.1007/s00284015-0947-y

35. Makarova KS, Aravind L, Wolf YI et al (2001) Genome of the extremely radiation-resistant bacterium Deinococcus radiodurans viewed from the perspective of comparative genomics. Microbiol Mol Biol Rev 65(1):44-79. doi:10.1128/MMBR.65.1.44-79.2001 
36. Oh D, Poulsen M, Currie C et al (2009) Dentigerumycin: a bacterial mediator of an ant-fungus symbiosis. Nat Chem Biol 5:391-393. doi:10.1038/nchembio.159

37. Russell JA, Moreau CS, Goldman-Huertas B et al (2009) Bacterial gut symbionts are tightly linked with the evolution of herbivory in ants. Proc Natl Acad Sci USA 106:21236-21241. doi:10.1073/pnas.0907926106

38. Ramalho MO, Bueno OC, Moreau CS (2017) Microbial composition of spiny ants (Hymenoptera: Formicidae: Polyrhachis) across their geographic range. BMC Evol Biol. doi:10.1186/ s12862-017-0945-8

39. Ramalho MO, Martins C, Silva LMR et al (2016) Molecular profile of the Brazilian Weaver Ant Camponotus textor Forel (Hymenoptera, Formicidae). Neotrop entomol. doi:10.1007/ s13744-016-0392-Z

40. Stadler B, Dixon AFG (2005) Ecology and evolution of aphid-ant interactions. Annu Rev Ecol Evol Syst 36:345-372. doi:10.1146/ annurev.ecolsys.36.091704.175531

41. Schultz TR, Brady SG (2008) Major evolutionary transitions in ant agriculture. Proc Natl Acad Sci USA 105:5435-5440. doi:10. 1073/pnas.0711024105

42. Sen R, Ishak H, Estrada D et al (2009) Generalized antifungal activity and screening of Pseudonocardia and Amycolatopsis bacteria in nests of fungus-growing ants. P Natl Acad Sci USA 106:17805-17810. doi:10.1073/pnas.0904827106

43. Scott JJ, Budsberg KJ, Suen G et al (2010) Microbial community structure of leaf-cutter ant fungus gardens and refuse dumps. PLoS ONE 5:e9922. doi:10.1371/journal.pone.0009922

44. Seipke RF, Barke J, Brearley C et al (2011) A single Streptomyces symbiont makes multiple antifungals to support the fungus farming ant Acromyrmex octospinosus. PLoS ONE 6:e22028. doi:10.1371/journal.pone.0022028

45. Seipke RF, Kaltenpoth M, Hutchings MI (2012) Streptomyces as symbionts: an emerging and widespread theme? FEMS Microbiol Rev 36:862-876. doi:10.1111/j.1574-6976.2011

46. Santos AV, Dillon RJ, Dillon VM et al (2004) Occurrence of the antibiotic producing bacterium Burkholderia sp. in colonies of the leaf-cutting ant Atta sexdens rubropilosa. FEMS Microbiol Lett 239:319-323. doi:10.1016/j.femsle.2004.09.005

47. Sapountzis P, Zhukova M, Hansen LH, Sørensen SJ, Schiøtt M, Boomsma JJ (2015) Acromyrmex leaf-cutting ants have simple gut microbiota with nitrogen-fixing potential. Appl Environ Microbiol 81:5527-5537. doi:10.1128/AEM.00961-15

48. Stoll S, Gadau J, Gross ROY et al (2007) Bacterial microbiota associated with ants of the genus Tetraponera. Biol J Linnean Soc 90:399-412. doi:10.1111/j.1095-8312.2006.00730.x

49. Sanders JG, Powell S, Kronauer DJ et al (2014) Stability and phylogenetic correlation in gut microbiota: lessons from ants and apes. Mol Ecol 23:1268-1283. doi:10.1111/mec.12611

50. Suen G, Scott JJ, Aylward FO et al (2010) An insect herbivore microbiome with high plant biomass-degrading capacity. PLoS Genet 6:9. doi:10.1371/journal.pgen.1001129

51. Thiaucourt F, Bolske G (1996) Contagious caprine pleuropneumonia and other pulmonary mycoplasmoses of sheep and goats. Rev Sci Tech 15:1397-1414

52. Vieira AS, Bueno OC, Camargo-Mathias MI (2012) Morphophysiological differences between the metapleural glands of fungus-growing and non-fungus growing ants (Hymenoptera, Formicidae). PLoS ONE 7:e43570. doi:10.1371/journal.pone. 0043570

53. Vieira AS, Morgan ED, Drijfhout FP et al (2012) Chemical composition of metapleural gland secretions of fungus-growing and non-fungus- growing ants. J Chem Ecol 38:1289e1297. doi:10.1007/s10886-012-0185-8

54. Vieira AS, Bueno OC (2017) Lipid mobilization and acyl-coenzyme A dehydrogenase abundance in the postpharyngeal gland of a leaf-cutting ant. Sociobiology 63(4):1005-1014. doi:10.13102/ sociobiology.v63i4.1027

55. Wertz JT, Kim E, Breznak JA et al (2012) Genomic and physiological characterization of the Verrucomicrobia isolate $\mathrm{Di}$ plosphaera colitermitum gen. nov., sp. nov., reveals microaerophily and nitrogen fixation genes. Appl Env Microbiol 78:1544-1555. doi:10.1128/AEM.06466-11

56. Zientz E, Feldhaar H, Stoll $S$ et al (2005) Insights into the microbial world associated with ants. Arch Microbiol 184:199-206. doi:10.1007/s00203-005-0041-0

57. Zucchi TD, Guidolin AS, Cônsoli FL (2010) Isolation and characterization of actinobacteria ectosymbionts from Acromyrmex subterraneus brunneus (Hymenoptera,Formicidae). Microbiol Res. doi:10.1016/j.micres.2010.01.009 